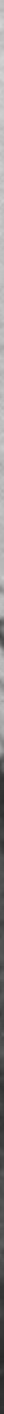

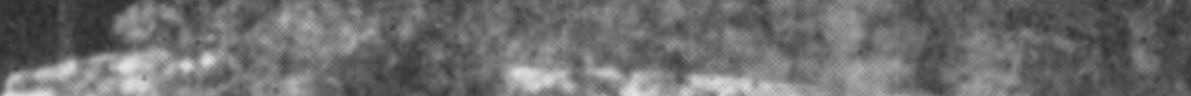

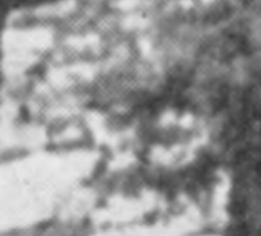

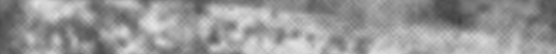

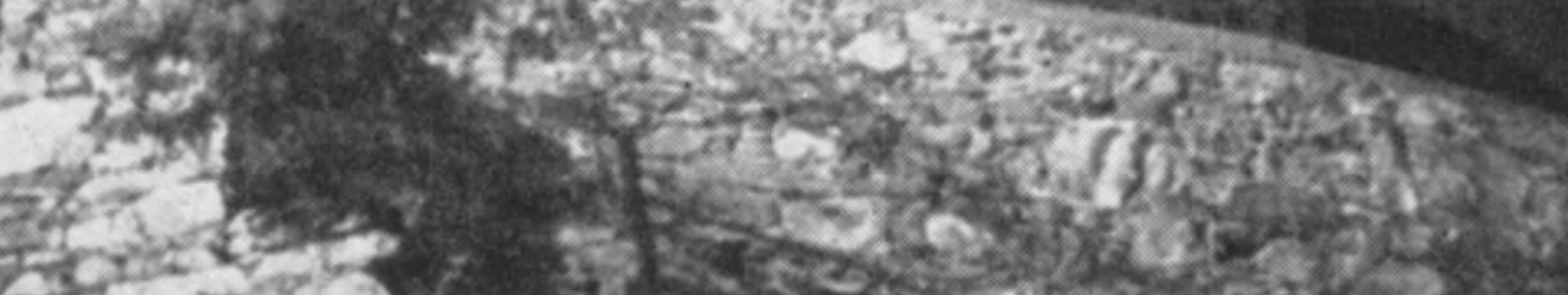

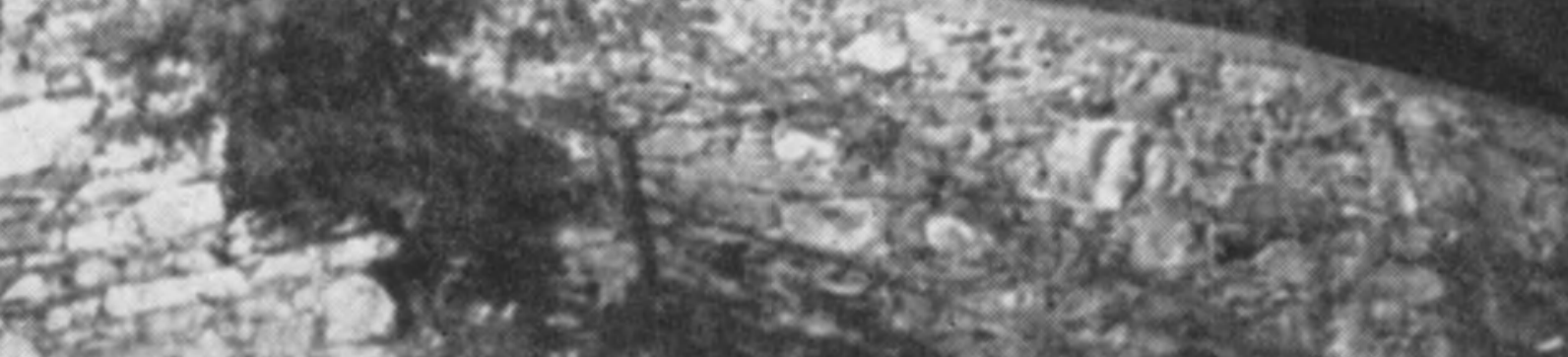

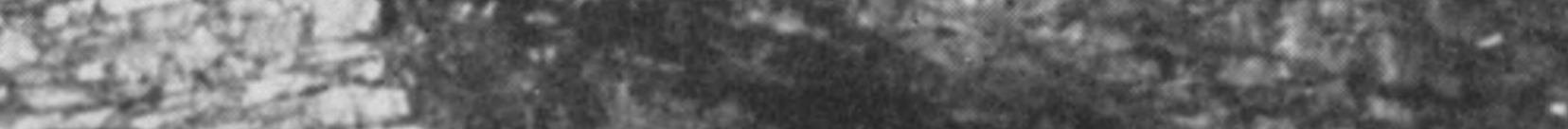

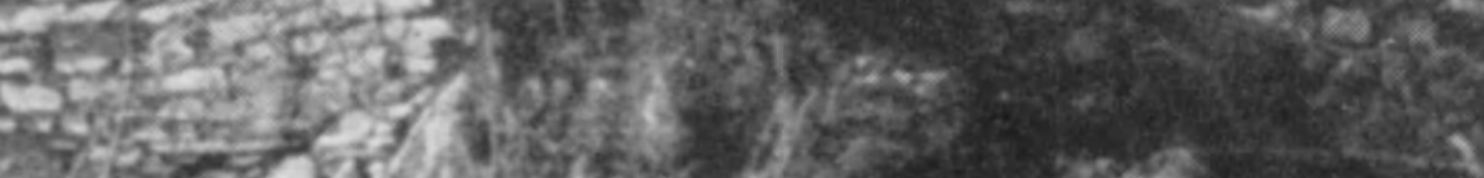

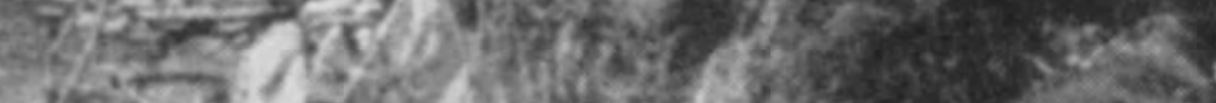

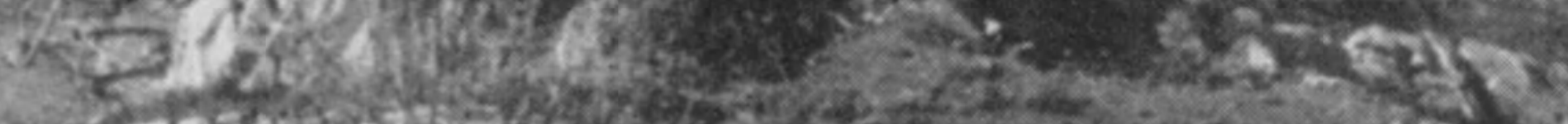

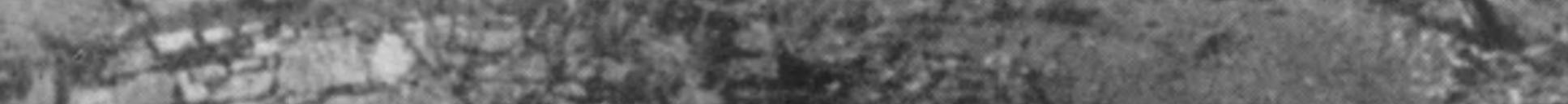




\title{
Geometria "imperfetta": Luigi Vietti, villa La Roccia a Cannobio
}

\author{
"Imperfect" geometry: Luigi Vietti, Villa La Roccia in Cannobio
}

\begin{abstract}
The article reviews the thought and work of Luigi Vietti (leading exponent of Italian Rationalism, then author of numerous domestic architectures, especially in Sardinia and on the Alps), through the presentation of one of his most important projects: the Villa La Roccia in Cannobio on the Lago Maggiore, completed in 1936. This project is based on a previous model, the "Villa su Roccia a Sperone", designed with a promotional target in mind. The latter was published in 1932 on «Domus Magazine» and was exposed in several Rational Architecture events. Between 1930 and 1936 he develops a new concept of architecture in relation to the site by means of topological reasoning. This article uses critical interpretation to highlight that, in Vietti's work, his interest in emerging architecture (shown by his participation in major founding events in the period between the Two World Wars) and the link to tradition, both contextual and disciplinary, manage to coexist in an often exemplary way. In Villa La Roccia, the character of the architecture as a whole and its details are remodeled to adapt to the rocky spur of Punta d'Amore. This makes the work better merge and blend with the surroundings. Even the interiors are recalibrated in relation to the site and domestic activities, emphasizing the precious definition of details and devoting particular attention to the perceptive-emotional factors of life within it.
\end{abstract}

\section{Paola Veronica Dell'Aira}

Architect, PhD, associate professor, full professor of Architectural and Urban Design, ICAR14, at Sapienza - Rome. Member of the Teaching Board of the Master in Lighting Design, and of the Doctorate in Architecture: Theories and Project. Among her major research interests: the theme of the relationship between uses and forms.
Keywords

Rationality, spontaneity, tradition, constructiveness, realism. 


\section{In coerenza con $i$ tempi}

A prima vista, il candore e la linearità del volume "quasi-cilindrico" tradiscono il desiderio di regolarità, essenzialità e astrazione che tanto impegnarono il "discorso" moderno. Anni Trenta, Luigi Vietti è tra le voci più attive. La regolarità era per sottrarsi all'arbitrio della scelta morfologica, l'essenzialità per rifuggire dall'eccesso di stile o, meglio, di neo-stile, l'astrazione per evitare il facile re-impiego di forme e modi bloccati da eccessivo ossequio verso il patrimonio disciplinare. Di motivi ce ne erano, pertanto. Lo stigma della storia gravava oltremodo sulla ricerca architettonica d'inizio secolo scorso: storia, non tradizione, non continuità diacronica, non aggiornamento, bensì, purtroppo, poca luce sul presente. Le ostinazioni formaliste di molti architetti di allora sollecitavano un'alternativa capace di produrre avanzamento. Occorreva un'opposizione forte, una vera e propria militanza. Le Corbusier aveva dato il via: quegli occhi... "non vedevano". Mentre l'ingegneria affinava le sue armi, abbracciando la sostanza costruttiva dei nuovi materiali e mezzi, relegando l'architettura a funzione accessoria: la superficie delle cose. "Attenzione signori architetti», avvisava il maestro nei suoi "trois rappels" di Vers une architecture. Tra i giovani del tempo, il fare squadra rivelava pertanto un'urgenza. Per alcuni, scoprì, miseramente, una scarsa vena inventiva. Per altri, la sfida fu grande: contrastare lo sterile manierismo in una presa di posizione forte. Contro lo spreco decorativo, serviva una dichiarazione di guerra: uno spazio cerebrale, ambienti come costrutti ideali, misura, standard, tecnicismo. L'adesione al Movimento Moderno era una sorta di atto d'obbligo. Ecco allora i Manifesti, i CIAM, il Werkbund e le Siedlungen, gli slogan. "La casa per tutti", il "form follows function", l'"existenzminimum"... L'Italia si schiera con gli obiettivi sovranazionali, sottoscrive l'universalismo della ricerca del tempo. Aderisce alle tesi e ai principi, si impegna nella manualistica, attraverso il CIRPAC (Comitato Internazionale per la Risoluzione dei Problemi dell'Architettura Contemporanea), è parte attiva nell'organizzazione dei Congressi. Nel 1927, Figini, Pollini, Frette, Larco, Rava, Terragni, Castagnoli, fondano il "Gruppo Sette", Libera si unisce all'équipe che si costituisce come MIAR (Movimen- to Italiano per l'Architettura Razionale). Luigi Vietti ne condivide gli assunti. Partecipa, in particolare, al $3^{\circ}$ Congresso CIAM (Bruxelles, 1930, tema: Rationelle Bebauungsweisen). Al MIAR però, non aderisce formalmente, pur curandone, con i colleghi, l'organizzazione; si dichiara e si espone, ma a modo suo; partecipa alla Prima Esposizione di Architettura Razionale (1928), alla Seconda (1931) e a quella di Firenze (1932); dal 1937, è poi coprogettista dell'EUR con Pagano, Piccinato, Rossi e Piacentini. I dubbi tuttavia non lo abbandonano; il rapporto con l'architettura "di regime" e, in particolare, la partnership con Piacentini, si rivelano faticosi. Strizzare l'occhio al potere, inseguire l'occasione di lavoro, gli valgono un buon primo piano. Ma condividerne a fondo i presupposti, gioire degli esiti, sono altra cosa. Punto di mediazione: Vietti si allinea, ma lasciando una porta aperta. È sensibile al calore dell'architettura spontanea, approfondisce i contesti, ama le difficoltà che ogni tema singolarmente pone, raccoglie, in particolare, la lezione che il diffcile territorio ligure, sua patria professionale, gli impartisce: terreno accidentato, fortemente dislivellato e compresso tra le Alpi e il mare. Al generalismo dei dettami ideologici e alla potenza dei simboli (non si sottrae infatti all'esplorazione di tipologie rappresentative, dalle Case del Fascio - Oleggio, Intra, Rapallo - al Concorso per il Palazzo Littorio di Roma) desidera affiancare una ricerca più puntuale e dedicata; alla retorica del discosto dal suolo, contrapporre il connubio, l'intreccio, l'ibridazione; alle premure figurative e di linguaggio far corrispondere la sostanza di uno spazio vissuto. Il tema dell'"abitare" domina e attraversa ogni spazio della sua produzione, sin da quel critico "squadrismo" d'ante-guerra, premonitore di un razionalismo destinato a far presto ritorno sui suoi passi, di un positivismo che diviene debole risorsa inventiva di fronte al vulcanico universo culturale della filosofia esistenzialista.

\section{Ancoraggio}

Villa La Roccia incarna una fede, senza farne oggetto di acritica assunzione. Della poetica di Vietti, rappresenta dunque un saggio esemplare. Dichiara l'architetto, in un'intervista rilasciata poco prima della sua scomparsa, di aver sempre creduto, fin dal- 
la propria tesi di laurea, nella conciliazione tra opposti, ossia nell'«accompagnamento delle due cose:

Fig. 1 razionalità e spontaneismo» e ne adduceva una dopL. Vietti, pia ragione: «gli elementi dell'architettura razionaInsediamento di Punta D'Amore a Cannobio, 1929 (CSAC, Parma).

Fig. 2 Veduta da sud-est (Architettura, a. XV, fasc. XII, dicembre

1936). lista erano diventati "fissi", gli architetti ci giocavano, in più pensavo a tutto il bagaglio di architettura spontanea del nostro paese [...]» (Dell'Aira, 1997). Nel n. 52/1932, della rivista «Domus», compare la presentazione di tre plastici di studio: "Tre ville progettate da Luigi Vietti". Colpisce la denominazione di ognuna, riferita univocamente, alla tipologia morfologica del territorio di riferimento: villa in pianu$\mathrm{ra}$, villa su terreno a fasce e villa su roccia a sperone.
Non serviva altro nome; non solo per l'inesistenza di committenze specifiche, ma anche e soprattutto per il loro mancare di una precisa localizzazione: tre modelli, pertanto, volutamente formulati come tali. Lungi però dal voler fare, del tema, astrazione, l'intenzione fu quella di condurre un ragionamento "esportabile", di mettere a punto strategie riferibili alle necessità insediative di terreni diversi, strategie chiare e precise, ma assolutamente non vincolanti e, quindi, flessibili, elastiche, trattabili.

C'è un sapere o, meglio, una teoria d'approccio, che inquadra bene quest'impegno. È la topologia. Il suo fondamento? Il "codice t.", ossia l'insieme dei segni
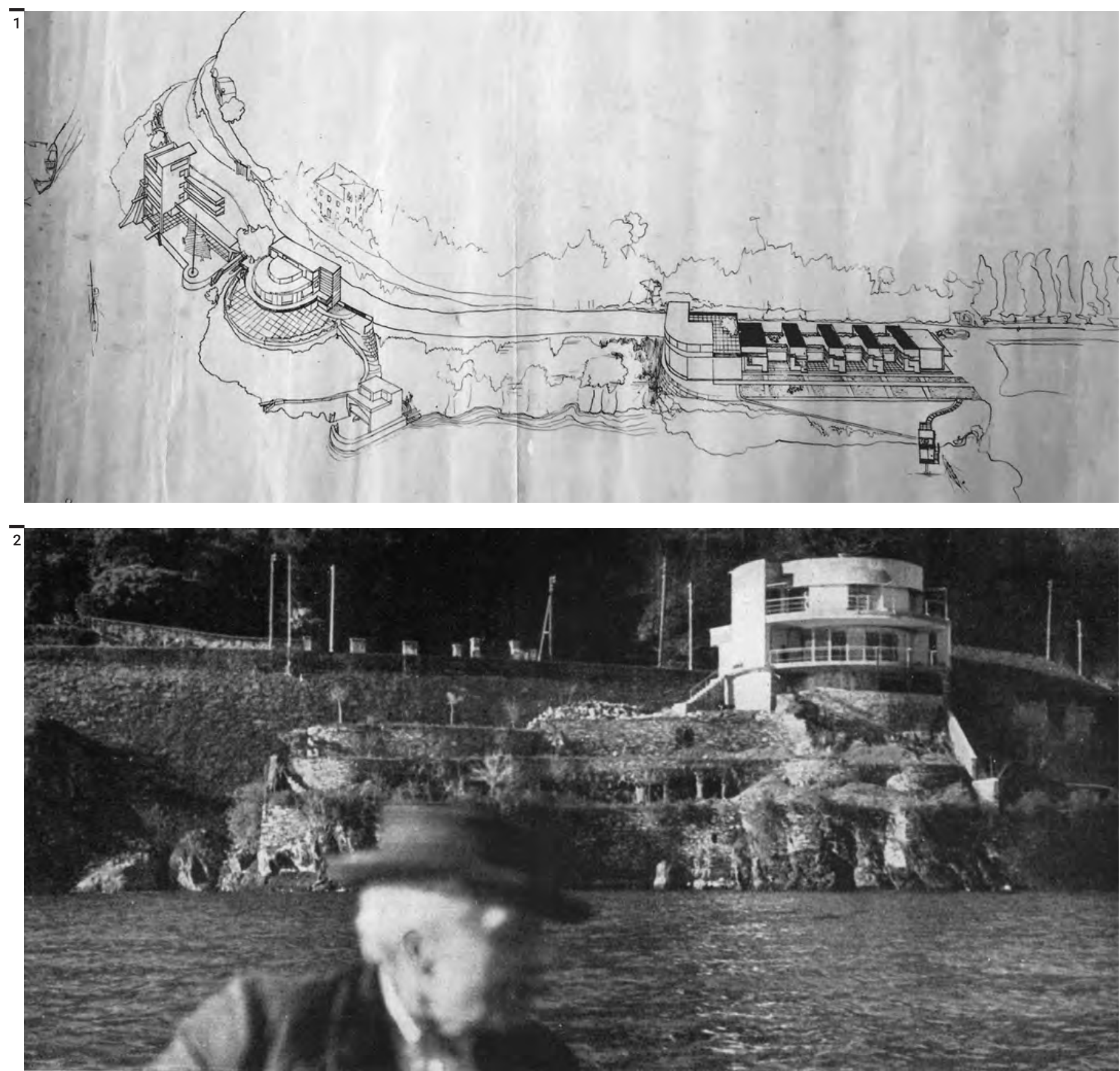


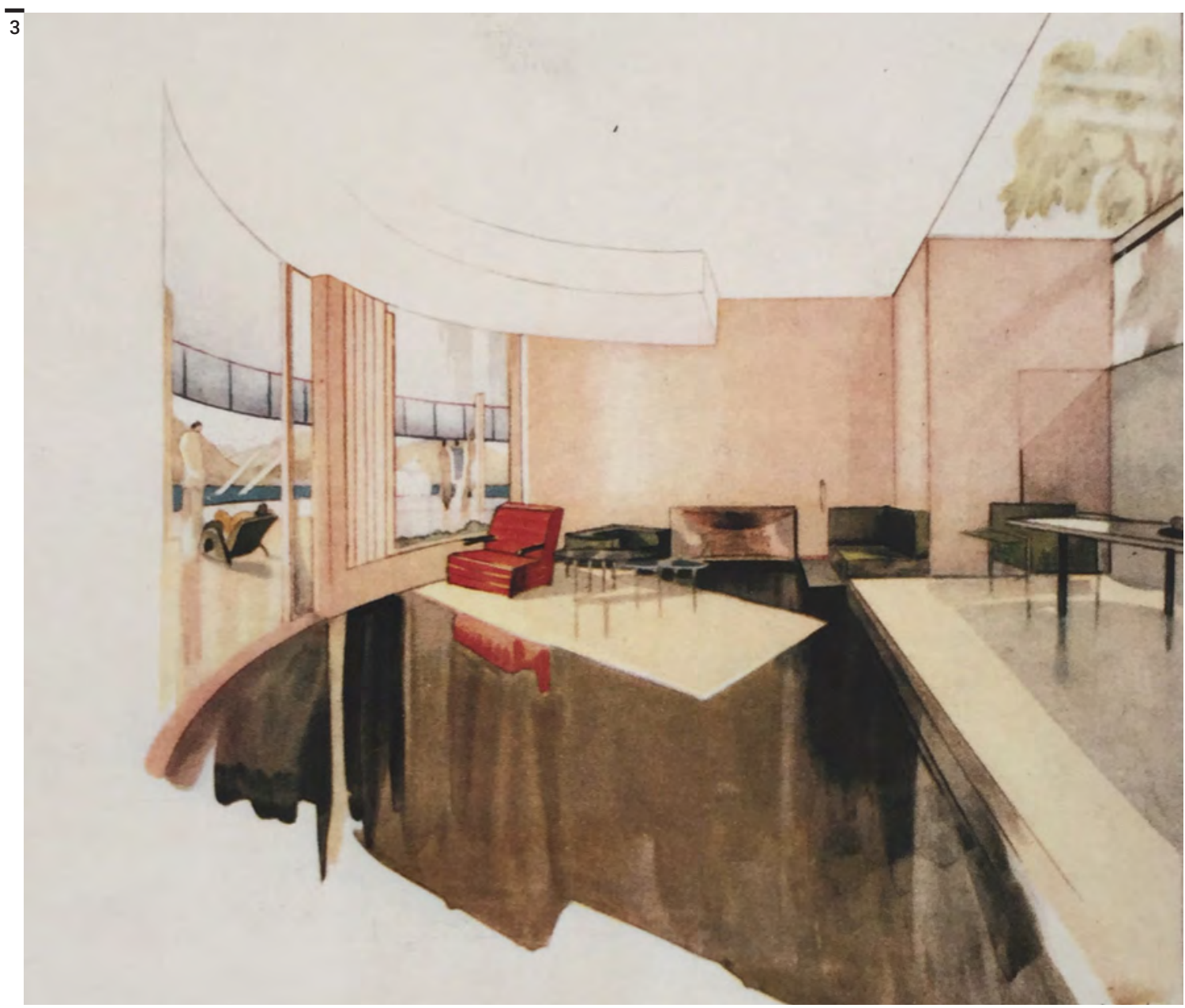

di cui la geografia si serve per rappresentare i vari tipi di forme del suolo, onde esprimere, sia i rapporti istituibili tra costruzione e sito di fondazione, sia gli indotti psicologico-percettivi derivanti dalla relazione tra soggetto e ambiente. Ulteriormente, la topologia osserva i luoghi in ordine alla loro trasformabilità: una trasformabilità, però, assolutamente custode delle caratteristiche originarie dei supporti orografici. La topologia è dunque, per l'intervento antropico, lo studio di figure e forme, volto a mantenerne le proprietà nell'incontro con il suolo. "Topologica" è una deformazione che opera senza "strappi", "sovrapposizioni" o "incollature" (Treccani, Wikipedia). Ora, i plastici pubblicati su «Domus», oggetto anche dei coevi eventi espositivi sull'architettura razionale, possono apparire di semplice promozione linguistica. Non vi è dubbio sull'interesse di Vietti per la forma razionale. Ma non è qui l'obiettivo. Sensibile ai concetti topologici di funzione continua, convergenza, continuità, connessione, Vietti ricerca soluzioni "di saldatura" con i luoghi. L'apparente astrazione teorica è tutt'altro che propaganda stilistica; a condurre è piuttosto l'idoneità ai luoghi, ancorché indagati in forma accorpata, ovvero per "famiglie" di situazioni: cuspidi, acclività, pianure, rispetto alle quali definire sviluppi distributivi, proporzioni, scelte posizionali, grandezze, esposizioni...

Sono dunque geometrie interpretabili, flessibili, territorialmente adattive. Non "fissano" la scelta. Piuttosto ne cercano, nel sito, la spinta immaginativa. La possibilità, nel legame, di trovare forme diverse. Caro infatti, alla topologia, è anche il concetto di varietà. Le geometrie di Vietti vogliono farsi contestuali... omeomorfiche.

Dei tre modelli, solo la villa su roccia a sperone, conosce però una completezza di percorso da "casa tipo" a "casa concreta". Intravede, infatti, sin dal principio, un'allocazione specifica. In quegli anni Vietti era impegnato in un progetto estensivo e complesso per la sistemazione del tratto di sponda del Lago Maggiore, detto della Punta di Amore in prossimità di Cannobio. In un disegno di Archivio (CSAG, Parma) compare una sequenza di volumi funzionalmente definiti e nominati in didascalia, distesi lungo il margine costiero (tra gli altri, un Bar Acqueo, un Ristorante, Darsene e Ville, dai nomi criptati di Dott. Z, sig. G.G., sig. Cocchio). Del bordo liminare, la villa su sperone, occupava la posizione più estrema ver- 


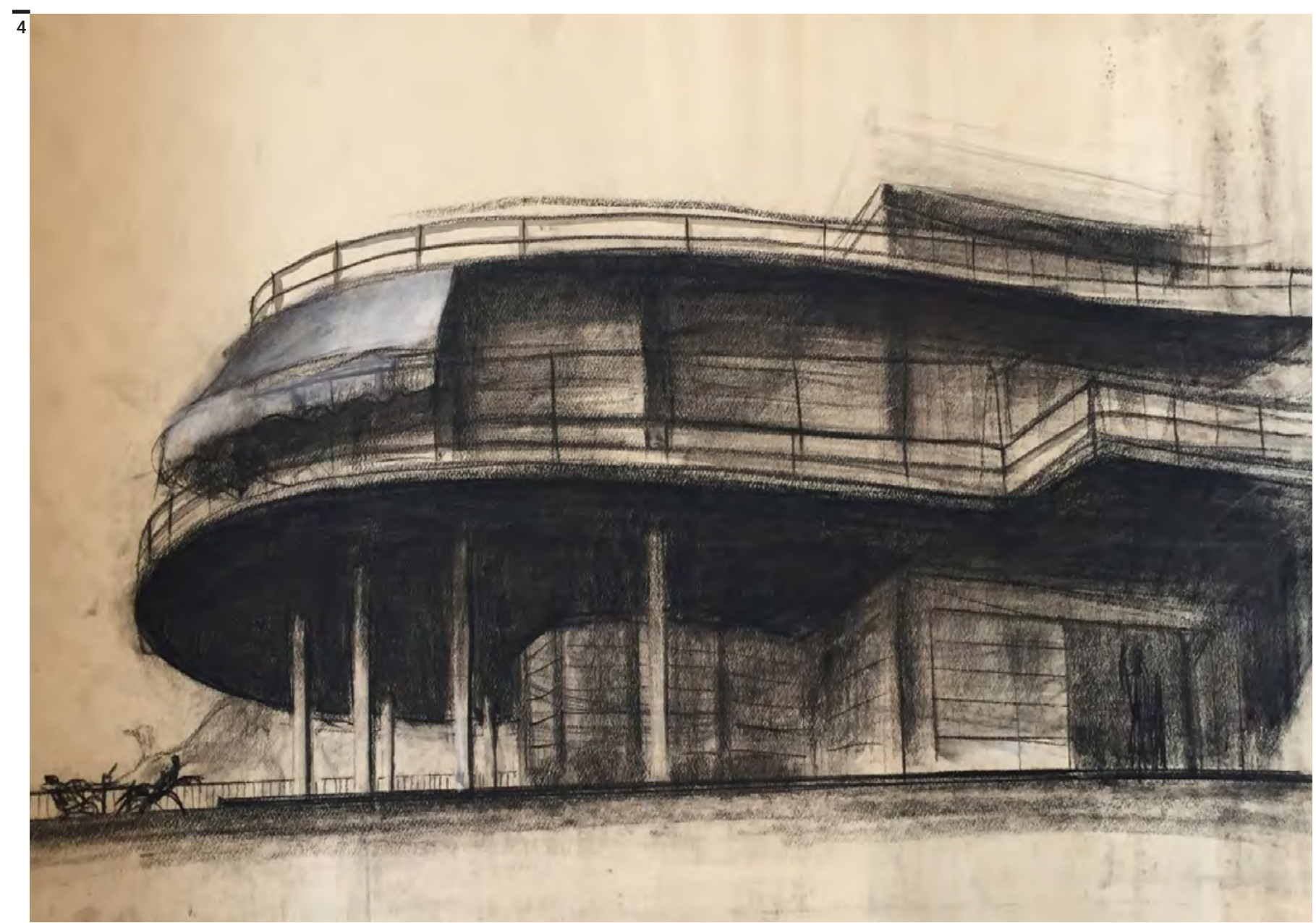

so lo specchio acqueo. Per massimizzare la panoramicità, Vietti utilizzava la disposizione a semicerchio: a un volume rettilineo che faceva da ingresso verso la strada, ne giustapponeva uno semicilindrico di due piani. Il primo piano, porticato, la zona living, si slanciava verso il lago con una profonda terrazza, anch'essa semicircolare; il secondo conteneva il "ventaglio" delle stanze da letto convergenti in una saletta per la prima colazione. È l'inizio di un viaggio, il crogiuolo di un'opera tutta ancora da con-formare.

\section{Casa d'abitazione}

La concrescenza tra la realizzata villa La Roccia e lo sperone roccioso che ancora la accoglie e, insieme, l'evoluzione avvenuta, a partire dal primo modello, tra 1930 e 1936, segnano un passaggio che fa scuola. «È una fondamentale visione del mondo - scrive Ch. Alexander in Pattern Language - essa ci dice che quando si costruisce una cosa, non si può semplicemente costruire quella cosa isolata, ma bisogna anche ri-

Fig. 4 Schizzo prospettico a carboncino (CSAC,

Parma). parare il mondo intorno ad essa e all'interno di essa, in modo che il mondo che la ingloba diventi più coerente e complesso, e la cosa che si realizza, così fatta, prenda il suo posto nella rete della natura».

La costruzione, che oggi apprezziamo, conserva assai poco dell'originaria soluzione. Nonostante le restrizioni imposte dal lotto effettivamente disponibile, non subisce diminuzioni qualitative, anzi. Più che occupare lo spazio, essa sembra attraversarlo, generando movimento, indicando direzioni, selezionando viste, recependo, dal sito, modifiche avvaloranti. Nel suo deformarsi in aderenza allo sperone, essa inoltre, più che togliere spazio, ne crea di ulteriore. Essa "fa spazio", come direbbe Merleau Ponty, invera il passaggio «de l'espace spatialisé à l'espace spatialisant» (Merleau Ponty, 1945).

Cura d'intorno, dunque, sensibilità dialogica, relazionalità, fisicità. Il volume si contrae per intensificare il legame di appartenenza allo sperone mentre, di quest'ultimo, si fa coronamento. Esso passa da due a tre livelli, fondendosi con la roccia sottostante attraverso un basamento in pietra. La terrazza arretra e si assottiglia; mantiene il suo andamento curvilineo, ma non è più esattamente un semi-cerchio; si interrompe e si raddrizza appoggiandosi, con una parete, alla roccia; si riduce anche in lunghezza, rivolgendosi maggiormente verso meridione. Il soggiorno, anch'esso, asseconda la torsione e la piega 


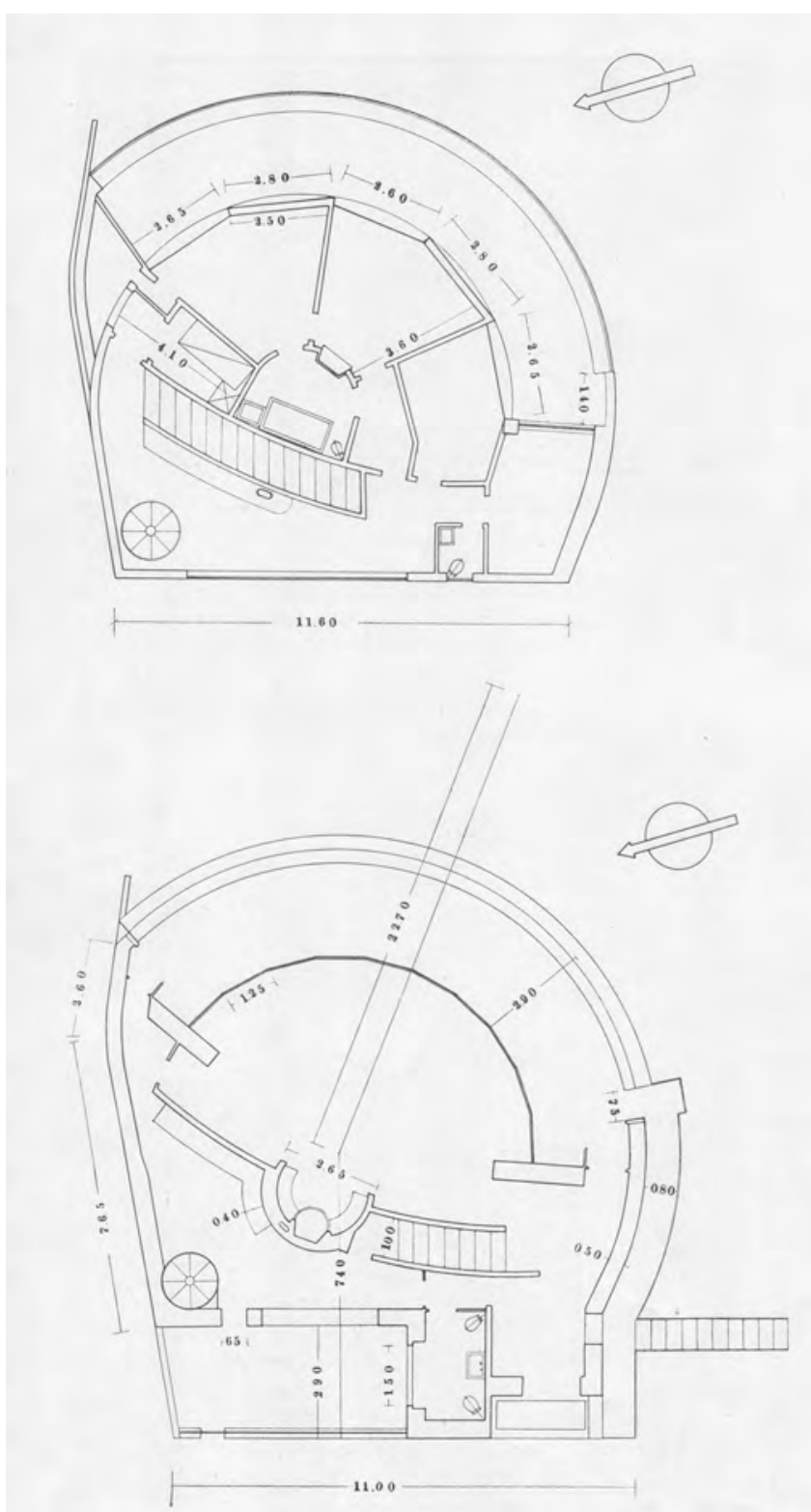

visuale della vetrata, mentre si chiude, a monte, con la nicchia del camino e la scala, anch'essa curvilinea. Le stanze, al piano superiore, passano da 4 a 3 , mentre si riducono gli spazi di distribuzione. Ora, la casa è più "misurata", ma non nel senso di una maggiore esattezza dimensionale e prestazionale, piuttosto nel suo esprimersi come spazio "leggibile", riconoscibile nelle sue proprietà qualitative più che quantitative, uno spazio non ideale, uno spazio che, prima di offrirsi alla vista, lascia intendere concrete possibilità d'uso. Abbandona la regolarità, l'omogeneità, l'isotropia della geometria euclidea. Ora, è spazio sensibile, anisotropo e disomogeneo. Il suo centro d'indagine non guarda più alle figure o alle forme, ma ai rapporti di posizione tra queste. Cresce inoltre, nella realizzazione, l'interesse per i fattori psicologico-emozionali che l'interno è capace di produrre in interazione con l'abitante.

Sì perché l'“abitare" non è solo nello star bene in un posto, nel contatto con il contesto ospitante; non è la semplice modellazione organica del confine tra spazio antropico e non (aspetto già presente, nella "villa modello", nel terrazzo forato per incorporare un albero preesistente). "Abitare", come in Heidegger, è l'esprimere vita, imprimendo, nello spazio, moventi d'uso, sia di carattere strettamente utilitario che di soddisfazione esperienziale, sensibile, emotiva (Heidegger, 1964).

Torna alla mente l'esperienza condotta da Bruno Taut con la costruzione della propria casa di Dahlewitz (1926), la cui volumetria complessiva mostrava la forma di un quarto di cerchio. I colleghi ne criticarono la sagoma "a formaggino", ironizzando sulla sua buffa iconicità. Per sfatare le illazioni, Bruno volle trasferire in un libro lo spirito delle sue scelte. Intitolò il testo Ein Whonhaus - Una casa d'abitazione (1927).

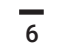

Fig. 5

Piante del primo e secondo piano (CSAC, Parma).

Fig. 6

Vista attuale $b / n$ (foto Alessandro Depaoli). 
L'intento era provocatorio: la casa è per viverci dentro. Un'ovvietà, si direbbe, una tautologia, con tanto di gioco di parole sul nome dell'autore. Cos'altro è la casa se non uno spazio da abitare? Cos'altro è se non uno spazio da definire attraverso lo sguardo, i sentimenti, la vita del suo abitante? Taut palesava, nel titolo, tutta la sua polemica nei confronti dello stigma modernista. La casa è da abitare, non da osservare. E così poco la osserva, Bruno, la sua casa, che omette di rappresentarla nel suo insieme. Dà priorità alle scene di vita interna. Quasi tutto il libro è infatti una sequenza di fotografie che documentano le attività domestiche, mentre ogni ambiente viene raffigurato singolarmente, disgiunto dalla sua appartenenza a una composizione complessiva. L'architettura è solo la cornice della scena. La sua forma non è nulla. Può anche essere fortemente iconica. Può anche avere un volume "a formaggino".

Così è per la villa di Cannobio: un cilindro inesatto, poiché la vita ha fatto ingresso al suo interno. $\mathrm{E}$ la vita vuole la quotidianità della scena, la sua familiare accoglienza; vuole semplicità costruttiva, laicità e indulgenza formale, mixité di materiali, vecchi e nuovi, motivi universalisti e/o autoctoni, disincanto linguistico. In villa La Roccia, c'è l'algida ringhiera in acciaio, il levigato intonaco, la copertura piana, il "pan de verre", ma non manca l'archetipo del camino, lo spessore delle pietre, il colore "tenue" della tinta ocra, il gradinare dei volumi, emulo dei terrazzamenti naturali sottostanti, dall'affondo nel suolo alla linea di gronda.

Come nella coeva villa Wanda di Stresa c'è una sensibilità moderna «non contaminata da teorie ne- bulose» (Vietti), poiché la "navalità" dei motivi figurativi è, anch'essa, in certa misura, un fattore locale, posizionate come sono, entrambe le ville, in un contesto di "montagna bassa", ovvero sul navigabile e teso pelo d'acqua del lago.

Vietti ci tiene ad affermare questo anti-ideologico approccio. "Non si capisce il tuo stile", gli criticavano i colleghi, "fai tutte cose diverse". Ma è proprio il difetto di incoerenza a svelare l'aspetto più durevole e istruttivo del suo lavoro: la tendenza ad azzerare di continuo il proprio repertorio espressivo. La villa La Roccia non è, per questo, né migliore né peggiore, rispetto al precedente modello "su roccia a sperone". Ne è, semplicemente, l'evoluzione, utile a sancire la bellezza dell'architettura come inarrestabile progress di arricchimento, frutto di un concept capace sempre di "mostrare il fianco". Nel 1930, la sua casa Il Ronco, a Pedemonte di Gravellona Toce, mostra una corda annodata, a far da mancorrente alla scala. E già, nei disegni della villa su roccia a sperone, la balaustra della scala, a dispetto dello stentoreo volume, si risolve in una rete di corda colorata. È la provvisorietà di "momenti non finiti" come direbbe Alvar Aalto, tali da alleviare l'effetto coercitivo degli stilemi; la retorica "purista", la patina e la fissità di soluzioni distanti dal cuore.

Si ringraziano Enrico Prandi, per il supporto e lo scambio teorico-critico sul tema, per la selezione archivistica dei disegni, dei documenti e delle foto dell'epoca; Riccardo Cattaneo Vietti, per l'interesse, la disponibilità, la fornitura di documentazione fotografica; Alessandro Depaoli, per la rassegna fotografica relativa allo stato attuale della villa.

\section{Bibliografia}

(1932), «Tre ville progettate da Luigi Vietti», in Domus, n. 52.

(1936), «La Roccia a Cannobio, arch. Luigi Vietti», in Architettura, Rivista del Sindacato Nazionale Fascista, Architetti, a. XV, fasc. XII, Milano.

(1937), «Villa La Roccia a Cannobio», in Casabella, n. 111.

Alexander Christopher (1977), A pattern language, Oxford University Press, New York.

Dell'Aira Paola Veronica (1997), Luigi Vietti. Progetti e realizzazioni degli anni '30, Alinea, Firenze.

Dell'Aira Paola Veronica (2019), «Attitudine creativa: Luigi Vietti», in FAM Magazine del Festival dell'Architettura. Ricerche e progetti sull'architettura e la città, n. 48.

Esposizione Internazionale Triennale di Monza (a cura di) (1936), 36 progetti di ville di architetti italiani, Ed. Triennale, Milano-Roma.

Heidegger Martin (1964) (trad. it. 2000), Corpo e spazio. Osservazioni su arte-scultura-spazio, II Melangolo, Genova. Merleau Ponty Maurice (1945), Phénoménologie de la perception, Gallimard, Paris.

Prandi Enrico (2019), «Prime indagini d'archivio sull'architettura di un maestro dimenticato», in FAM Magazine del Festival dell'Architettura. Ricerche e progetti sull'architettura e la città, n. 48.

Taut Bruno (1927), Ein Whonhaus, in Salotti Gian Domenico (a cura di) (1986), Bruno Taut, Una casa di abitazione, Franco Angeli, Milano.

\section{Fonti}

Archivio CSAC (Centro Studi e Archivio della Comunicazione - Università di Parma), Fondo Luigi Vietti - Co-direzione Enrico Prandi, Paola V. Dell'Aira.

Convegno-Seminario di Studi "Lezioni Italiane. Gardella, Menghi, Vietti", Parma, Abbazia di Valserena, 1516/03/2019, a cura di C. Quintelli, A. Lorenzi, E. Prandi, C. Gandolfi.

Ricerca Interateneo "Luigi Vietti e il professionismo italiano (1928-1998)" in corso al CSAC di Parma, Responsabile Scientifico E. Prandi. 